\title{
AC 2010-26: IMPROVING TEAM PERFORMANCE: THE COGNITIVE STYLE FACTOR
}

\section{Joanna DeFranco, Pennsylvania State University}

Joanna F. DeFranco is an Engineering faculty member at Penn State University. She earned her B.S. in Electrical Engineering and Math from Penn State, M.S. in Computer Engineering from Villanova, and earned her Ph.D. in Computer and Information science from New Jersey Institute of Technology. Previous to entering academia, Dr. DeFranco held a number of positions in industry and government, including software engineer for Motorola in Horsham, PA and an Electronics Engineer for the Naval Air Development Center in Warminster, PA. She has published a number of articles in journals and conference proceedings in the area of collaborative problem solving, group cognition, global engineering, and computer forensics.

\section{Colin Neill, Pennsylvania State University}

Colin J. Neill, associate professor of software and systems engineering and associate division head of engineering and information science, earned his Ph.D. in software and systems engineering, M.Sc. in communication systems, and B.Eng. in electrical and electronic engineering from the University of Wales, Swansea, United Kingdom. He teaches in the graduate systems engineering, software engineering, and engineering management programs. Prior to joining Penn State, Dr. Neill worked on manufacturing and enterprise systems with Oxford University, the Rover Car Company, and British Aerospace. His research has been funded by the Ben Franklin Technology Partners and the Department of Defense. He has written more than sixty articles on software design, architecture, process, and management, and serves as associate editor-in-chief of Innovations in Software and Systems Engineering. 


\title{
Improving Team Performance: The Cognitive Style Factor
}

\begin{abstract}
It is widely considered that success in the design and development of an engineering system is contingent upon the team having a shared vision of the problem they are solving. The goal of this research is to determine which factors improve the performance of an engineering team. One of the aspects explored is the effectiveness of arranging teams based upon each team member's cognitive problem solving style preference using the Adaption-Innovation framework ${ }^{1}$. This paper presents a complete experiment evaluating concept map data from the design stage of engineering, graduate student, teams.
\end{abstract}

\section{Introduction}

In previous research we showed that the Cognitive Collaborative Model (CCM) can improve team performance in systems design ${ }^{2}$ and may also be effective in facilitating a shared vision, or mental model of the problem being solved by a team ${ }^{3}$. Research indicates, however, that working within a team actually generates its own set of problems: the difficulties associated with managing the diversity of those within a team, referred to as Problem B (in contrast to Problem A: solving the actual problem on which the team is working) ${ }^{4}$.

Diversity here refers to the difference in problem solving style preferences of the individuals comprising the team. In the A-I framework, one's problem-solving preference reveals how one visualizes, conceptualizes, and communicates about the problem the team is attempting to solve. An individual's preference is at a point along a continuum from more adaptive to more innovative. A more adaptive problem solver seeks to refine or improve upon existing solutions whereas more innovative problem solvers propose to alter solutions outside those already proposed, and possibly outside the existing paradigm. In a randomly assigned team we could reasonably expect dispersion across that continuum and the theory implies that the dissonance between individuals distant along the continuum will lead to Problem B.

To investigate the impact of problem-solving diversity we used the A-I cognitive style inventory to determine the problem solving styles of individuals working in a team and measured the degree of convergence of the team's mental model. Concept maps were used to elicit and represent team member mental models and an application called Pathfinder Network analysis along with a descriptive evaluation were used to determine the degree of commonality and similarity within each team.

\section{Background}

Cooke et al. [2001] investigated the factors that influence team performance and found that both taskwork knowledge (i.e., understanding of the task at hand) and team situational awareness (i.e., understanding of a complex and dynamic situation at any one point in time) were good predictors of team performance. This implies that team cognition is an important factor in the success of a 
team. Indeed, "each person's ability to function successfully depends upon coordinated cognitive interactions with others, and the cognitive 'products' that emerge from these interactions cannot be attributed to single individuals," that "they detect and recognize pertinent cues, make decisions, solve problems, remember relevant information, plan, acquire knowledge, and design solutions or products as an integrated unit." ". This is not to say, however, that team cognition is a natural outcome of working on a team. In fact, it requires a lot of effort and is a distinguishing feature of high-performance teams 8 . Team members come with differing perspectives, backgrounds, and expectations and from this diversity a shared mental model must emerge?

An individual's mental model is an "internal conceptual representation of an external system whose structure maintains the perceived structure of that system" 10 . In plainer terms, we "understand the world by constructing working models of it in [our] minds", . But when we work in teams our mental models must be consistent, or at least we should collectively form a new mental model, a shared or team mental model, in order to experience the team cognition and its accompanying advances in efficiency and effectiveness. The phenomena of shared mental models and their formation have been studied by many. Mitchell [1986] refers to them as shared internal frames of reference, Walsh et al. [1988] investigated them as negotiated belief structures, and Rentsch and Hall [1994] examined team member schema similarity. Moreover, numerous studies have confirmed the impact on team effectiveness of a consistent shared/team mental $\operatorname{model}^{12,13,14,15}$.

While there is consensus on the importance and impact of shared mental models, there is little consensus on how to elicit and represent those models. Mohammed, Kilmoski, and Rentsch [2000] provide a detailed survey of the literature on team mental models, and reveal that many methods exist for eliciting mental models including similarity ratings, concept mapping, card sorting, and various questionnaires. Carley [1997] automatically generated individual and shared mental models using textual analysis. A technique that has been successfully employed by Clariana [2009a; 2009b].

There is likewise a variety of representation tools including Pathfinder networks ${ }^{19,20}$, concept and cognitive maps ${ }^{17,18}$, and multi-dimensional scaling ${ }^{15,16}$. Similarly, there are several recognized techniques for analyzing the resultant mental models and measuring the degree of similarity and convergence. Langan-Fox et al. [2000] surveyed those analytical techniques and recommended using Pathfinder Network Analysis to compare concept maps, and the NETSIM function to measure the degree of similarity within a team's mental models. This is the approach used in this study and has been successfully employed in several others ${ }^{14,19,20}$.

Now, the majority of studies into team cognition presume heterogeneity with respect to the backgrounds and worldviews of the team members, but homogeneity of individuals' cognitive processes. Kirton [2006] posits, however, that everyone's cognitive style sits along a continuum from more adaptive to more innovative. Where one falls on the continuum relates to ones preference in dealing with structure. For example, a more adaptive problem solver sees structure as enabling and a more innovative problem solver sees structure as limiting. Having a structure become tighter, sharper, clearer can be very positive, however, it may be tough to include something new. Kirton calls this the paradox of structure and we must ensure that any 
collaborative model does not fall victim to it by ensuring that it is sufficiently flexible to allow each team member to express their thinking, while sufficiently predictable so that each member can structure their understanding. The structure can be rules that surround the problem, solution, or process as well as the paradigm in which the problem/solution exists. Put simply, a more adaptive person prefers to change a structure by making that process more efficient and a more innovative person prefers to make that process different. Not one style is better than another. We need a diverse set of problem solvers to solve a diverse set of problems. Managing the diversity is part of the problem solving challenge. This research places attention on the problem solver and their precise effect on the problem solving process.

\section{Theory}

\section{Adaption Innovation}

AI theory is founded on the assumption that a person's level (ability, IQ, experience) and their style (their approach to, or preference in, problem solving) are not correlated. Often without knowledge of cognitive style diversity, a style difference may be misinterpreted as a level difference between two problem solvers. This is because level differences are easier to understand. This obviously adds to Problem B thus getting in the way of solving Problem A since it is clearly easier to get along with someone like us. To be effective, the team must expend more energy on Problem A than Problem B. The teams will typically manage this by introducing structure to their thinking, their interactions, and their communications. Unfortunately, this structure is simultaneously enabling (in that it provides a framework for cognition, for example in a taxonomy), and limiting (in that it constrains the available options, for example when we experience a paradigm shift).

More adaptive problem solvers tend to accept a problem as it is defined and tend to agree on the general constraints of the problem. Immediate increased efficiency is a high priority for a more adaptive problem solver. A problem solver that is more innovative may reject and redefine the constraints and generally accepted perception of the problem. They are less concerned with immediate efficiency and are more concerned with long-term gains ${ }^{1}$.

Problem B, usually caused by the style diversity of the team can also be defined as "cognitive gap". We need to figure out how we close that gap so the team has a full understanding of Problem A. The CCM could be part of the answer.

\section{$C C M$}

The main goal of the CCM is to assist in facilitating critical thinking and effective problem solving among the collaborators. The CCM described briefly in this paper is made up of six stages: Problem Formulation, Solution Planning, Solution Design, Solution Translation, Solution Testing, and Solution Delivery. Each stage is further broken down into three phases.

For the purposes of this paper we will only focus on the details of the first two stages of the CCM: problem formulation and solution planning. The three phases of the problem formulation stage (stage 1) are: Preliminary Problem Description, Preliminary Mental Model, and Structured 
Problem Representation. The goal of this stage is for the team to answer questions and gather information for each team member to understand the problem. In addition, the individuals need to communicate effectively and the group also needs to listen and make sure each member has the correct understanding of the problem.

For example, in the first stage the collaborators are to agree upon a preliminary problem description to make sure each team member has the same understanding of the problem. The model guides each team member to create a description in their own words and share it to each team member. Each team member discusses and votes to determine one problem description. Next, the team is charged with answering questions to develop a preliminary mental model. For example, the questions help the team to discus and determine givens, unknowns, conditions and constraints on the problem. The final part of this stage is for the team to identify and organize any relevant information to the problem thus creating a knowledge base from which the team will begin their solution plan (stage 2). The team organizes the information from the preliminary mental model by using the suggested format.

The three phases of the second stage of the CCM, solution planning, are: Strategy Discovery, Goal Decomposition, and Data Modeling. The goal for this stage of the CCM is for the team to answer questions and gather information to plan a solution. Specifically, they are going through the process of goal decomposition where they are refining goals into smaller sub-goals that are more easily solved. In addition, this stage provides a scheme to organize related data is discussed by the team.

For example, in the initial phase of stage two the team is beginning to strategize solution alternatives. The model provides questions for each team member to critically think about the solution alternatives. The team members then share their solution possibilities with each other. A vote commences to determine the solution that will be followed. Now that the team has agreed upon a solution it can be broken down into sub goals and distributed among the team members. The final phase of stage two is to yield a preliminary data model. This is accomplished by each team member associating their sub goal with the facts from the output of stage one. Specifically, they are integrating the givens and unknowns from stage one with the refined goals of stage two.

In the remaining four stages of the CCM the team would be translating the plan into a detailed design, implementing the design, testing, and finally delivering the solution. Working through the first two stages of the CCM, the team is able to conceptualize the problem resulting in a more effective plan and in theory implementing a better solution.

\section{Concept map analysis}

It has been determined that the CCM results in an improved outcome in the teams utilizing the model $^{2}$. The hypothesis as to why the CCM teams performed better is that these teams increased collaboration resulted in a convergent mental model of the project in which they were working. We used concept maps to qualitatively and quantitatively measure the convergence of the mental models. While there are many techniques for eliciting and analyzing tacit mental models few offer the advantages of concept mapping. Concept maps are intuitive and therefore do not require 
substantial training and only brief instructions, and they support multiple analysis techniques, many of which are automated, thus allowing larger numbers of participants in studies and less opportunity for investigator biases. The complete concept map evaluation is accomplished in three main dimensions that are discussed briefly below and in more detail in the results section of this paper.

1. Overlap: Defined as the analysis of the overlap of terms between team members. Concept maps can be constructed in two ways: where each individual constructs the map from concepts he/she identifies (open map), or when each map is constructed from a predetermined list of concepts agreed upon by the team or specified by the investigator (closed map). Closed maps are easier to analyze but they are restrictive to only the concepts available. Open maps are more difficult to analyze but allows for freedom to determine the concepts. This study used open maps. Having an overlap of concepts implies having a similar language about the problem which can increase communication later in the project development. The following are the two ways we evaluate the overlap:

a. Overlaps: count the number of terms similar in all maps

b. Overlap similarity: calculate a percentage of overlap similarity=(overlap average)/(total concepts)

2. Link: Links are the lines drawn between two concepts in a map. Evaluations of the links between common terms are evaluated using Link Similarity and Map Density.

a. Link similarity: (shared links between maps)/(union of all links)

b. Map density (concept to link ratio): (\# of concepts)/(total links)

3. Distance: Evaluation of relative proximity of common terms. It is the actual pixel distance between common terms. The students were instructed that related terms should be put closer together. The shortest path between common terms was determined by Pathfinder. Pathfinder creates links if two terms that are relatively close to one another. Therefore, the distance similarity equation looks similar to the link similarity equation.

a. Distance similarity: (shared links relatively close to one another)/(union of all links)

The results of the analysis techniques will be discussed in the next section as well as what the results of each part of the analysis reveals about team mental model convergence and how the $\mathrm{CCM}$ and team member cognitive style may have influenced those results.

\section{Hypothesis, Methodology, and Results}

This pilot study involved 15 experienced engineers randomly divided into five teams of three. Three of the teams were instructed to use the CCM during the design phase of their project. Each team completed a different project of equal complexity. The KAI inventory was utilized to determine where the participants fell on the AI continuum. Table 1 shows the team AI average score. The diversity was determined by averaging the differential between team member's AI scores. Any team that averaged more than 10 points differential is considered diverse. Despite the teams being randomly formed, each condition (CCM, no CCM) had both cognitively 
homogeneous and diverse teams. According to AI theory diversity is good, as long as the team can manage it otherwise this creates problem B. A difference of 10 points between two people is noticeable over time; 20 points or more can lead to communication problems. As shown in Table 1, team 3 and especially team 4 have more than a 20 point differential.

Table 1: AI Team Average

\begin{tabular}{|l|l|l|l|l|}
\hline Team & AI Average & Diverse? & Model & $\begin{array}{l}\text { Average } \\
\text { differential }\end{array}$ \\
\hline 1 & 91 & Yes & No CCM & 15.3 \\
\hline 2 & 99.67 & No & No CCM & 7.33 \\
\hline 3 & 95 & Yes & CCM & 23.3 \\
\hline 4 & 84.3 & Yes & CCM & 34.6 \\
\hline 5 & 99.3 & No & CCM & 8.66 \\
\hline
\end{tabular}

Each team was asked to individually create a concept map of their project in the middle of the semester. This was to ensure the design portion of the project was complete as well as having any cognitive differences time to take effect.

As described previously there are three quantitative dimensions to concept maps evaluation, overlap, link and distance analyses. However, it is not meaningful to do significance testing on these figures due to the small $\mathrm{N}$ and the fact that the teams worked on different projects. It is meaningful, however, to look for a pattern within the data and perform a more descriptive analysis of the teams in addition to a quantitative analysis.

\section{Overlap}

Table 2 shows the overlap data. The average number of terms the team members had in common with each other. The hypothesis is that a cognitively diverse team utilizing the CCM will have the most overlap - which is the case. The team not utilizing the CCM as well as having very similar cognitive styles to each other would have the least overlap - which is also the case. To be complete we also calculated the similarity overlap using the following equation:

\section{Overlap similarity $=($ average overlap $) /($ average $\#$ of terms $)$}

Noting the worst team (overlap of 3 and overlap similarity of 12.6\%) did not have access to the CCM and also did not have cognitive diversity. The other teams did not vary greatly when comparing the overlap similarity. Note that team 4 with the highest average overlap had a lower overlap similarity than their condition counterparts this is where the large AI differential may have had an effect.

Table 2: Overlap Analysis 


\begin{tabular}{|l|l|l|l|l|l|}
\hline Team & $\begin{array}{l}\text { Avg. } \\
\text { Overlap }\end{array}$ & $\begin{array}{l}\text { Avg. \# } \\
\text { of terms }\end{array}$ & Group & \multicolumn{1}{|c|}{ AI } & $\begin{array}{c}\text { Overlap } \\
\text { similarity }\end{array}$ \\
\hline 1 & 8 & 13.3 & No CCM & Diverse & $60 \%$ \\
\hline 2 & 3 & 23.67 & No CCM & Homogeneous & $12.6 \%$ \\
\hline 3 & 10 & 16.67 & CCM & Diverse & $59.8 \%$ \\
\hline 4 & 12.7 & 27.33 & CCM & Diverse & $46.4 \%$ \\
\hline 5 & 7 & 12.67 & CCM & Homogeneous & $55 \%$ \\
\hline
\end{tabular}

\section{Link}

Two different link analysis techniques were performed. The first technique is a link analysis using Pathfinder to calculate map similarity. The second technique is using the links to calculate map density. In the first technique, Pathfinder compares the maps and calculates the total number of derived links shared between the team maps (the intersection of the maps). This value is referred to as common and ranges from 0 to the maximum number of links.

Finally, Pathfinder calculates the "similarity" values for each pair of maps by dividing the common value by the union of the two maps - the total number of links. Similarity can range between 0 and 1 with smaller values indicating weaker relationships between the maps.

A limitation of the Pathfinder link analysis is that it will not include any indirect links. For example, if in map 1 we have A- B and in Map 2 we have A-C-B, these two maps will not have a link in common even though A and B are clearly linked. Thus, stressing the importance of also analyzing the map density (concept to link ratio).

Table 3 shows the results from the Pathfinder link analysis. The data shows higher map similarity in the cognitively diverse teams. In particular, teams 1 and 3 had the highest scores with team 3 having scores that are most similar to each other. This similarity of scores indicates better communication by the team. The following is a descriptive analysis summary explaining that claim:

\section{Team1:}

No CCM - AI Diverse (15.3 differential) - Similarity scores not similar

A smaller AI differential requires less effort to communicate effectively. The smaller differential should have worked to their benefit. CCM could have helped the team make the best use of their style diversity.

One team member's similarity score stands out - that team member has the highest differential AI score. This adds support to the descriptive analysis

\section{Team 3:}


The moderate AI differential required more effort to communicate effectively which was accomplished in this team (as compared to team 1). The CCM may have helped this team make best use of their moderate style diversity.

\section{Team 4:}

\section{CCM - AI Diverse (34.6 differential) - similarity scores not similar}

This AI differential is extremely significant. The team member that stands out has the highest differential scores (52 points from one member and 39 points from another). This person actually submitted three concept maps which seemed to depict each team members part of the project - confirming the disconnect within the team. Again, adding support to this descriptive analysis that the large differential is difficult to manage even with the CCM.

Table 3: Link Analysis

\begin{tabular}{|l|l|l|l|}
\hline Team & Similarity & \multicolumn{1}{c|}{ Group } & \multicolumn{1}{c|}{ AI } \\
\hline 1 & .55 & No CCM & Diverse \\
\hline 1 & .18 & No CCM & Diverse \\
\hline 1 & .7 & No CCM & Diverse \\
\hline 2 & 0 & No CCM & Homogeneous \\
\hline 2 & 0 & No CCM & Homogeneous \\
\hline 2 & 0 & No CCM & Homogeneous \\
\hline 3 & .29 & CCM & Diverse \\
\hline 3 & .26 & CCM & Diverse \\
\hline 3 & .23 & CCM & Diverse \\
\hline 4 & .17 & CCM & Diverse \\
\hline 4 & .09 & CCM & Diverse \\
\hline 4 & .09 & CCM & Diverse \\
\hline 5 & 0 & CCM & Homogeneous \\
\hline 5 & .06 & CCM & Homogeneous \\
\hline 5 & .1 & CCM & Homogeneous \\
\hline
\end{tabular}

The second type of link analysis is calculating the map density. Density of a map shows higher order thinking 5 . Map density is a concept to link ratio. Table 4 shows the concept to link ratio for each team as well as their individual and average team AI scores. A lower ratio indicates a more dense network of concepts relating to higher evaluation and synthesis among concepts. The best team (lowest ratio) was team 3 which was a cognitively diverse ( 23 point differential) CCM team.

The overall density mean for diverse teams was .73 with a standard deviation of .05 . The mean for the homogeneously AI teams was .88 with a standard deviation of 0.1 . The density mean for the CCM/non CCM teams was virtually the same. This could be explained by the much larger AI differential within team 4. Looking further to explain this theory, team 1 and 4 had almost 
the same mean density score, notice that the score skewing the average for team 4 is the person with the 52 and 39 point differential with their other team members. As previously mentioned, this may have caused communication, mutual respect issues. Without the team members having insight into their cognitive diversity this gap may not have been managed with the needed skill and coping behavior. Team 1 had one person also skewing the score, however, there team differential was more than half of team 4's differential - again working to their benefit.

Table 4: Map Density

\begin{tabular}{|c|c|c|c|c|}
\hline Team & concepts & Links & Concept:Link & Al score \\
\hline $1 a$ & 18 & 26 & 0.692307692 & 78 \\
\hline $1 b$ & 13 & 18 & 0.722222222 & 101 \\
\hline $1 c$ & 9 & 11 & 0.818181818 & 94 \\
\hline average & 0.744237 & & & 91 \\
\hline stdev & 0.065761 & & & \\
\hline $2 a$ & 17 & 22 & 0.772727273 & 98 \\
\hline $2 b$ & 16 & 20 & 0.8 & 95 \\
\hline $2 c$ & 39 & 46 & 0.847826087 & 106 \\
\hline average & 0.806851 & & & 99.66667 \\
\hline stdev & 0.038015 & & & \\
\hline $3 a$ & 19 & 27 & 0.703703704 & 110 \\
\hline $3 b$ & 24 & 37 & 0.648648649 & 100 \\
\hline $3 c$ & 9 & 13 & 0.692307692 & 75 \\
\hline average & 0.681553 & & & 95 \\
\hline stdev & 0.02906 & & & \\
\hline $4 a$ & 14 & 15 & 0.933333333 & 54 \\
\hline $4 b$ & 27 & 37 & 0.72972973 & 93 \\
\hline $4 c$ & 31 & 45 & 0.688888889 & 106 \\
\hline average & 0.783984 & & & 84.33333 \\
\hline stdev & 0.130942 & & & \\
\hline $5 a$ & 11 & 10 & 1.1 & 106 \\
\hline $5 b$ & 19 & 20 & 0.95 & 93 \\
\hline $5 c$ & 9 & 11 & 0.818181818 & 99 \\
\hline average & 0.956061 & & & 99.33333 \\
\hline stdev & 0.141007 & & & \\
\hline
\end{tabular}

Cognitive diversity and the CCM had a positive effect on the teams, to summarize our findings so far,

1. The cognitively diverse team having a moderate KAI differential (23.3) AND access to the CCM has closer similarity scores (depth) and high overlap score (breadth). 
2. The cognitively diverse team having a large KAI differential (34.6) AND access to the CCM had excellent breadth (concept overlap) however, had a communication problem with map depth.

3. The cognitively diverse team having a small KAI differential (15.3) AND no access to the CCM had a communication problem with map breadth (map overlap) and depth (link).

4. The cognitively homogeneous teams did the worst. The team without access to the CCM in last place with the CCM team slightly ahead.

This, with additional data in another experiment could show that the CCM assists in collaborating a project breadth (as shown with the concept overlap analysis- team 4 with the largest differential had the most overlap), however, if the KAI differential is very large, map density/links may suffer since the team may be not have easily communicated depth of the project.

\section{Distance}

The distance analysis, shown in Table 5, evaluates the proximity between common terms (concepts). This type of analysis also has limitations because it is only reliable if the number of terms in the comparison maps is similar. For example, if map A is larger (has more terms) but the same number of common terms as map B. Map B will have a better distance score since the common terms are obviously closer together. Again, since these maps were all of different projects it important to look at all of the metrics associated with this part of the analysis: similarity scores, standard deviation of the scores, and the average number of terms per team.

Table 5: Distance Analysis

\begin{tabular}{|c|c|c|c|c|c|c|c|}
\hline Team & Similarity & $\begin{array}{l}\text { Team } \\
\text { mean }\end{array}$ & $\begin{array}{c}\text { Team } \\
\text { Standard } \\
\text { Deviation }\end{array}$ & $\begin{array}{l}\text { Number } \\
\text { of terms }\end{array}$ & $\begin{array}{l}\text { Average } \\
\text { number } \\
\text { of terms }\end{array}$ & Group & $\mathbf{A I}$ \\
\hline 1 & .29 & & & 18 & & $\begin{array}{l}\text { No } \\
\text { CCM }\end{array}$ & Diverse \\
\hline 1 & .18 & & & 13 & & $\begin{array}{l}\mathrm{No} \\
\mathrm{CCM}\end{array}$ & Diverse \\
\hline 1 & .39 & .29 & .11 & 9 & 13.3 & $\begin{array}{l}\text { No } \\
\text { CCM }\end{array}$ & Diverse \\
\hline 2 & 0.71 & & & 17 & & $\begin{array}{l}\text { No } \\
\text { CCM }\end{array}$ & Homogeneous \\
\hline 2 & .2 & & & 16 & & $\begin{array}{l}\text { No } \\
\text { CCM }\end{array}$ & Homogeneous \\
\hline 2 & .17 & .36 & .30 & 38 & 23.7 & $\begin{array}{l}\text { No } \\
\text { CCM }\end{array}$ & Homogeneous \\
\hline 3 & .18 & & & 19 & & $\mathrm{CCM}$ & Diverse \\
\hline 3 & .23 & & & 22 & & $\mathrm{CCM}$ & Diverse \\
\hline 3 & .25 & .22 & .04 & 9 & 16.7 & $\mathrm{CCM}$ & Diverse \\
\hline
\end{tabular}




\begin{tabular}{|l|l|l|l|l|l|l|l|}
\hline 4 & .2 & & & 24 & & CCM & Diverse \\
\hline 4 & .11 & & & 27 & & CCM & Diverse \\
\hline 4 & .13 & .15 & .05 & 31 & 27.3 & CCM & Diverse \\
\hline 5 & .13 & & & 10 & & CCM & Homogeneous \\
\hline 5 & .46 & & & 19 & & CCM & Homogeneous \\
\hline 5 & .13 & .24 & .19 & 9 & 12.7 & CCM & Homogeneous \\
\hline
\end{tabular}

The team with the highest similarity mean is team 2 - the worst team in all other analyses (overlaps and link) - highlighting the downside of this type of analysis. However, if we look at all the measures: team similarity mean, standard deviation of the mean, average number of terms, the best teams are teams 3 and 4 (CCM and cognitively diverse) - the similarity average are lower than the rest but they have a small standard deviation and a high number of average terms.

\section{Conclusions and Future Work}

This research intends to determine factors that improve efficiency within a team. In order to promote the factors that improve collaboration to students; we need to understand all of the factors that play a part in collaboration. We know from our previous research that the CCM has a positive impact on the teams who use it during the design stages of a project. We also know that each problem solver on a team has their own problem solving style preference which clearly is a factor in collaborative problem solving. Our goal was to determine what type of effect varying cognitive style also has on a problem solving team.

The main focus of this paper is evaluating the cognitive style effect. In addition to gathering insight into what is going on in a team and insight into what a team needs to be successful. We did this by evaluating each team's mental model of their projects to determine which combination of cognitive styles had the most convergent mental model. The teams created concept maps to depict their mental model of their project.

From this pilot study we have found support that cognitively diverse team's who have access to a collaborative cognitive model generated concept maps that are quantitatively and qualitatively more convergent than those without access to such models. This indicates that the CCM aides the team in managing their diversity (Problem B). This is encouraging since cognitive diversity within a team is desirable with respect to the solving of the initial problem (Problem A) provided that diversity does not distract the focus and energy of the team. Our next step has three dimensions: to repeat this experiment with a larger sample in order to conduct significance tests to confirm or refute our hypotheses, determine if concept map convergence is evidence of team learning, and if improved team cognition lead to improved learning of the individuals.

\section{Bibliography}

1. Kirton, M., Adaption-Innovation: In the context of Diversity and Change, Routledge 2006.

2. J.F. DeFranco and C.J. Neill. (2009) Improving Learning Outcomes using Cognitive Models in Systems Design, Proc. ASEE Annual Conference, Austin, TX, June 2009 
3. J.F. DeFranco, C.J. Neill, and R.B. Clariana. (2009) A Cognitive Collaborative Model to Improve Team Performance - A Study of Team Outcomes and Mental Model Sharing. In submission, Systems Engineering, 2010. 4. K.W. Jablokow. Developing Problem Solving Leadership: A Cognitive Approach. International Journal of Engineering Education, Volume 24, Number 5, September 2008, pp. 936-95.

5. Walker, J.M.T., King, P.H., “Concept Mapping as a Form of Student Assessment and Instruction”, Proceedings of the American Society for Engineering Education Annual Conference, 2002.

6. J.M. Levine, L.V. Resnick, and E.T. Higgins. (1993) Social foundations of cognition. Annual Review of Psychology, Vol. 44, January 1993, pp. 585-612.

7. N.J. Cooke, E. Salas, P.A. Kiekel, and B. Bell. (2004). Advances in measuring team cognition. In E. Salas \& S. M. Fiore (Eds.), Team cognition: Understanding the factors that drive process and performance (pp. 83-106). Washington, DC: American Psychological Association.

8. R. Stout, J.A. Cannon-Bowers, and E. Salas. (1996). The role of shared mental models in developing team situation awareness: Implications for training. Training Research Journal, 2, 85-116.

9. L.L. Levesque, J.M. Wilson, and D.R. Wholey. (2001) Cognitive Divergence and Shared Mental Models in Software Development Project Teams. Journal of Organizational Behavior, Vol. 22, No. 2, March, 2001, pp. 135144

10. J.K. Doyle, and D.N. Ford. (1998). Mental models concepts for system dynamics research. System Dynamics Review: The Journal of the System Dynamics Society, Vol. 14, No. 1, Spring 1998, pp. 3-30.

11. P.N. Johnson-Laird. (1983). Mental models: Toward a cognitive science of language, inference, and consciousness. Cambridge, MA: Harvard University Press.

12. J.A. Cannon-Bowers, E. Salas, and S.A. Converse. (1993). Shared mental models in expert team decision making. In N. J. Castellan, Jr. (Ed.), Current issues in individual and group decision making (pp. 221-246).

Hillsdale, N J: Erlbaum.

13. R. Klimoski and S. Mohammed. (1994). Team mental model: Construct or metaphor? Journal of Management, 20, 403-437.

14. J.E. Mathieu, G.F. Goodwin, T.S. Heffner, E. Salas and J.A. Cannon-Bowers. (2002) The Influence of Shared Mental Models on Team Process and Performance. Journal of Applied Psychology, 2000, Vol. 85, No. 2, pp. $273-$ 283.

15. J.R. Rentsch and R.J. Klimoski. (2001) Why Do 'Great Minds' Think Alike?: Antecedents of Team Member Schema Agreement. Journal of Organizational Behavior, Vol. 22, No. 2, March, 2001, pp. 107-120.

16. J.R. Rentsch. (1993) Predicting team effectiveness from teamwork schema similarity. Paper presented at the annual meeting of the Academy of Management, Atlanta, GA, 1993.

17. M.J. Kolkman, M. Kok, A. van der Veen. (2005) Mental model mapping as a new tool to analyze the use of information in decision-making in integrated water management. Physics and Chemistry of the Earth, Vol. 30, Iss 45, 2005, pp. 317-332.

18. K.M. Carley. (1997) Extracting Team Mental Models through Textual Analysis. Journal of Organizational Behavior Special Issue: Computers Can Read as Well as Count: Computer-Aided Text Analysis in Organizational Research, Vol. 18, November 1997. pp. 533-558.

19. N.J. Cooke, E. Salas, J.A. Cannon-Bowers, and R.J. Stout. (2000) Measuring Team Knowledge. Human Factors, Vol. 42, No. 1, Spring 2000, pp. 151-173.

20. R.B. Clariana, (2009 est.). Deriving group knowledge structure from semantic maps and from essays. In D. Ifenthaler, P. Pirnay-Dummer, \& N.M. Seel (Eds.), Computer-Based Diagnostics and Systematic Analysis of Knowledge (pp. xx-xx). New York, NY: Springer.

21. R.B. Clariana. (2009 est.). Pair-wise, list-wise, and clustering approaches for eliciting knowledge structure. In D. Ifenthaler, P. Pirnay-Dummer, \& N.M. Seel (Eds.), Computer-Based Diagnostics and Systematic Analysis of Knowledge (pp. xx-xx). New York, NY: Springer.

22. N.J. Cooke, P.A. Kiekel, and E. Helm. (2001). Measuring team knowledge during skill acquisition of a complex task. International Journal of Cognitive Ergonomics: Special Section on Knowledge Acquisition, Vol. 5, Iss. 3, September 2001, pp. 297-315.

23. R. Mitchell. (1986) Team building by disclosure of internal frames of reference. Journal of Applied Behavioral Science, Vol. 22, No. 1, 1986, pp. 15-28.

24. J.P. Walsh, C.M. Henderson, and J. Deighton. (1988) Negotiated belief structures and decision performance: an empirical investigation. Organizational Behavior and Human Decision Processes, Vol. 42, Iss. 2, October 1988, pp. 194-216. 
25. J.R. Rentsch and R.J. Hall. (1994) Members of Great Teams Think Alike: A Model of the Effectiveness and Schema Similarity Among Team Members. In Advances in Interdisciplinary Studies of Work Teams, Vol. 1. Series on Self- Managed Work Teams. M.M. Beyerlein, D.A. Johnson DA (eds). JAI Press: Greenwich, CT, pp, $223-262$. 26. S. Mohammed, R.Klimoski and J.R. Rentsch. (2000) The Measurement of Team Mental Models: We Have No Shared Schema. Organizational Research Methods, Vol. 3 No. 2, April 2000, pp. 123-165.

27. J. Langan-Fox, S. Code and K Langfield-Smith. (2000) Team Mental Models: Techniques, Methods, and Analytic Approaches. Human Factors, Vol. 42, No. 2, Summer 2000, pp. 242-271. 